\title{
Quarenteners vs. Cloroquiners: a framework to analyze the effect of political polarization on social distance stances
}

\author{
Régis Ebeling, Carlos Abel Córdova Sáenz, Jeferson Nobre, Karin Becker \\ Universidade Federal do Rio Grande do Sul, Brazil \\ \{rebeling, cacsaenz, jcnobre, karin.becker\}@inf.ufrgs.br
}

\begin{abstract}
The worldwide COVID-19 pandemic has struck people's lives overnight. With an alarming contagious rate and no effective treatments or vaccines, it has evoked all sorts of reactions. In this paper, we propose a framework to analyze how political polarization affects groups' behavior with opposed stances, using the Brazilian COVID polarized scenario as a case study. Two Twitter groups represent the pro/against social isolation stances referred to as Chloroquiners and Quarenteners. The framework encompasses: a) techniques to automatically infer from users political orientation, b) topic modeling to discover the homogeneity of concerns expressed by each group; c) network analysis and community detection to characterize their behavior as a social network group and d) analysis of linguistic characteristics to identify psychological aspects. Our main findings confirm that Cloroquiners are right-wing partisans, whereas Quarenteners are more related to the left-wing. The political polarization of Chloroquiners and Quarenteners influence the arguments of economy and life, and support/opposition to the president. As a group, the network of Chloroquiners is more closed and connected, and Quarenteners have a more diverse political engagement. In terms of psychological aspects, polarized groups come together on cognitive issues and negative emotions.
\end{abstract}

CCS Concepts: • Human-centered computing $\rightarrow$ Collaborative and social computing design and evaluation methods.

Keywords: political polarization, COVID-19, analysis framework, group behavior

\section{INTRODUÇÃO}

O Brasil vive um cenário político polarizado desde as eleições de 2014 que resultaram em um impeachment da então presidente. As eleições de 2018 dividiram ainda mais a população, fazendo que muitos votassem de acordo com sua convicção de direita/esquerda, ou qualquer coisa oposta a seus representantes (anti-Bolsonaro ou anti-PT). Neste cenário ainda polarizado, com apoios e críticas ao atual governo, instala-se a pandemia mundial da COVID-19, com consequências à saúde e à economia.

A COVID suscitou outra polarização no Brasil, quando se passou a discutir a prioridade entre saúde e economia. Pautados pela ciência e experiências de outros países, a medida inicial do Ministério da Saúde foi centrada em isolamento social, a qual foi adotada pela maioria dos governadores. Por outro lado, o presidente, eleito em parte por uma população ávida por melhorias econômicas, questiona o impacto destas medidas na economia, e defende medidas mais brandas, como a cloroquina. Este embate levou inclusive à demissão do Ministro da Saúde. Este cenário de oposição passa a ser representado por dois grandes movimentos pró/contra isolamento, denominados vulgarmente como "Quarenteners" e "Cloroquiners". Nas redes sociais, os pontos de vista dos dois movimentos passam a ser expressos massivamente, utilizando hashtags como \#OBrasilNãoPodeParar ou \#OBrasilTemQuePararBolsonaro.

Existem poucos trabalhos formalmente publicados investigando a COVID nas redes sociais. A maioria aborda modelagem de tópicos e modelos de difusão relacionados à COVID, conforme compilação apresentada em [Ordun et al. 2020]. Em relação à polarização política, [Sha et al. 2020] analisa as narrativas do Twitter em torno dos governadores dos EUA e da tomada de decisões presidenciais. Propriedades linguísticas foram aproveitadas para caracterizar a polarização política na postura de

Esta pesquisa é apoiada pela FAPERGS (projeto 19/2551-0001862-2).

Copyright(C)2020 Permission to copy without fee all or part of the material printed in KDMiLe is granted provided that the copies are not made or distributed for commercial advantage, and that notice is given that copying is by permission of the Sociedade Brasileira de Computação. 
tiroteios em massa [Demszky et al. 2019], violência racial [De Choudhury et al. 2016] e aspectos psicológicos dos políticos [Slatcher et al. 2007].

Neste artigo, propomos um framework para analisar como a polarização política afeta o comportamento de grupos com posicionamentos opostos no cenário brasileiro da COVID. Os posicionamentos pró/contra o isolamento social são representados por dois grupos no Twitter denominados Cloroquiners e Quarenteners, e seus comportamentos são comparados a um grupo sem motivações políticas. O framework engloba: a) técnicas para inferir automaticamente a orientação política dos usuários; b) modelagem de tópicos para descobrir as preocupações expressas por cada grupo; c) análise de rede e detecção de comunidades para caracterizar seu comportamento como grupo de rede social, e d) análise das características linguísticas para identificar aspectos psicológicos. Embora o framework de análise se concentre na COVID, ele poderia ser empregado para entender o impacto da polarização política nos posicionamentos que atualmente dividem ainda mais a população, como mudanças climáticas ou proteção ao meio ambiente. No cenário brasileiro da COVID-19, buscamos responder as perguntas: Q1) Há diferença nos assuntos comentados dentro de cada movimento?; Q2) Há diferenças na polarização política dentre os movimentos? e Q3) Os movimentos apresentam aspectos psicológicos distintos?

Nossos principais achados confirmam que a polarização política dos Cloroquiners e Quarenteners influencia os argumentos de economia e vida, e de apoio/oposição ao presidente. Como grupo, a rede dos Cloroquiners é mais fechada e conectada, enquanto os Quarenteners têm um envolvimento político mais diverso. Em termos de aspectos psicológicos, esses grupos compartilham traços comuns como aspectos cognitivos e emoções negativas.

O restante deste trabalho está estruturado como segue. A Seção 2 discute os trabalhos relacionados. A Seção 3 discute os dados e o método de análise proposto. A Seção 4 apresenta o resultado das análises visando responder às questões de pesquisa. A Seção 5 apresenta conclusões e trabalhos futuros.

\section{TRABALHOS RELACIONADOS}

O Twitter vem sendo utilizado para estudo de diferentes fenômenos sociais, tais como reações emocionais a traumas coletivos [Harb et al. 2019], equidade racial [De Choudhury et al. 2016] ou polarização política [Garimella and Weber 2017; Demszky et al. 2019]. Informações extraídas automaticamente dos perfis permitem aprofundar a compreensão destes fenômenos, tais como a orientação política de um usuário baseada nos políticos que segue [Garimella and Weber 2017].

A análise automática de aspectos linguísticos usando o LIWC (Linguistic Inquiry and Word Count $)^{1}$ tem embasado a caracterização de diferentes aspectos psicológicos [Tausczik and Pennebaker 2010]. LIWC categoriza as palavras em classes/subclasses de acordo com 4 dimensões linguísticas: processos linguísticos (e.g. pronomes), processos psicológicos (e.g. sociais, cognitivos), preocupações pessoais (e.g. trabalho, lazer) e linguagem informal (e.g. palavras de concordância).Tipicamente busca-se caracterizar aspectos psicológicos baseado no uso diferenciado destas palavras. [Demszky et al. 2019] analisa estados afetivos e uso de pronomes em tweets relacionados a tiroteios em massa, mostrando que a polarização política afeta a reação a estes eventos. Aspectos psicológicas como Honestidade e Complexidade Cognitiva são caracterizados em [Slatcher et al. 2007] para candidatos à presidência dos EUA usando combinações de classes linguísticas extraídas de discursos e debates. Analisando tweets com classes do LIWC, [De Choudhury et al. 2016] revela que estados dos EUA com alto índice de violência racial estão associados a negatividade, baixa cognição e baixa consciência social.

No alcance de nosso conhecimento, não existem trabalhos formalmente publicados que relacionam a polarização política e a COVID. Estudos preliminares [Pennycook et al. 2020] baseados em entrevistas mostram que percepções equivocadas sobre a pandemia da COVID são afetadas em maior grau pela baixa sofisticação cognitiva do que razões ideológicas. Uma revisão de relatórios técnicos que analisam tweets sobre a COVID é apresentada em [Ordun et al. 2020], mostrando que boa parte dos trabalhos se concentra na descoberta de tópicos e criação de modelos de difusão. Analisando os tópicos de

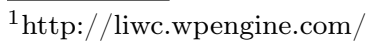


tweets sobre COVID, [Sha et al. 2020] desenvolve um modelo de narrativa, mostrando a influência do governo e suas secretarias na discussão de tópicos.

O presente trabalho contribui com um framework unificado, que abrange diferentes dimensões de análise para a compreensão dos efeitos da polarização no Twitter, aplicando-o ao cenário brasileiro da COVID. Além da modelagem de tópicos, é explorada a mensuração e caracterização da polarização política dos grupos, bem como aspectos psicológicos que podem ser inferidos de padrões linguísticos.

\section{FRAMEWORK DE ANÁLISE}

Propomos um framework para analisar os efeitos da polarização política sobre o comportamento de grupos com posicionamentos opostos, usando o cenário polarizado da COVID brasileiro como estudo de caso. O framework engloba técnicas para caracterizar o comportamento dos grupos segundo múltiplas dimensões: a) preocupações e assuntos comentados, explorando modelagem de tópicos e categorização linguística, (b) propriedades de redes sociais, analisando o índice de polarização política dos mesmos e detecção das comunidades polarizadas, e (c) aspectos psicológicos utilizando categorização linguística. A aplicação das técnicas associadas a cada dimensão de análise são detalhadas no restante desta seção.

\subsection{Dados}

Para investigar o comportamento polarizado pró e contra isolamento, analisamos características de três movimentos ligados a medidas de isolamento social adotadas em razão da pandemia da COVID, representados pelas hashtags presentes nos trending topics do Twitter ao final de março de 2020.

- Cloroquiners: para representar este movimento, escolhemos a hashtag \#OBrasilNãoPodeParar, referente à campanha de mesmo nome lançada pelo governo federal em 27 de março de 2020 . A campanha argumenta que o isolamento social traz efeitos mais devastadores à população pelas consequências à economia. Foram coletados 74.395 tweets de 20.572 usuários;

- Quarenteners: para capturar uma reação polarizada, identificamos a hashtag \#OBrasilTemQuePararBolsonaro $^{2}$, oposição à campanha governamental, com 10.769 usuários e 31.060 tweets;

- Neutros: como os dois movimentos acima têm claramente um viés político, procurou-se também movimentos surgidos antes deles, representados pelas hashtags \#FiqueEmCasa e \#FicaEmCasa. É observado que o foco maior deste grupo é no isolamento, a priori sem polarização política. O movimento ocasionou 201.499 tweets e 102.309 usuários.

A coleta de tweets e perfis de usuários envolvidos foi realizada com a API GetOldTweets ${ }^{3}$, que possibilita a captação de tweets antigos. A coleta ocorreu entre 22 de março de 2020 a 07 de abril de 2020, período em que foi observada uma maior utilização destas hashtags. Os tweets coletados estão disponibilizados em um repositório público ${ }^{4}$. Utilizamos a API Botometer ${ }^{5}$ para eliminar os bots, e então foi realizado um pré processamento para as etapas de análises. Aplicamos a normalização em letras minúsculas, remoção de stopwords, pontuação, caracteres especiais, hashtags, urls e menções a outros usuários. Descartamos também os tweets com menos de 3 termos.

\subsection{Caracterização de Tópicos}

Para identificar os assuntos discutidos por cada movimento estudado, utilizamos o modelo de tópicos Latent Direchlet Allocation (LDA) [Blei et al. 2003], que tem como entrada um corpus e um parâmetro $k$ que corresponde à quantidade de tópicos a serem descobertos. A saída é um conjunto de $k$ tópicos, cada qual composto por termos e a respectiva probabilidade beta de pertencerem ao tópico, e uma probabilidade gamma de relação de cada tópico com cada documento do corpus. A métrica CV [Röder et al. 2015] permite avaliar a coerência dos tópicos resultantes segundo múltiplas dimensões.

\footnotetext{
${ }^{2}$ https://getdaytrends.com/trend/\%23OBrasilTemQuePararBolsonaro/

${ }^{3}$ https://github.com/Jefferson-Henrique/GetOldTweets-python

${ }^{4}$ https://github.com/regisebeling/Quarenteners_Cloroquiners

${ }^{5}$ https://rapidapi.com/OSoMe/api/botometer-pro
} 
Aplicamos o LDA sobre o conjunto de tweets pré-processados de cada movimento. Para encontrar o melhor $k$ de cada grupo, variamos o mesmo de 1 a 30, e selecionamos os melhores valores de coerência utilizando a métrica CV. Então inspecionamos os resultados manualmente, utilizando os termos mais representativos de cada tópico, e uma amostra de tweets associados, verificando como interpretá-los. O $k$ escolhido em cada caso representa o menor conjunto de tópicos encontrados, cada qual com um conjunto coerente de termos, e com a menor redundância de assuntos. Para os Cloroquiners e Quarenteners foi escolhido $k=3$, e para Neutros, $k=8$, além de utilizar alpha $=0,5$ e beta $=$ auto.

\subsection{Análise da Polarização Política}

Usando os usuários seguidos (followings) dos usuários de cada movimento, verificamos a polarização política segundo dois métodos: cálculo de uma medida de proximidade de políticos de esquerda/direita [Garimella and Weber 2017] e análise dos grafos de seguimento. Dada a infraestrutura computacional disponível durante a pandemia para coleta dos followings, esta análise foi executada sobre uma amostra aleatória dos dados. Foram coletadas a lista de followings de $8.724(42,40 \%)$ usuários dos Cloroquiners, $4.553(42,27 \%)$ usuários dos Quarenteners e 8.361 (8,17\%) dos Neutros.

A lista de políticos foi estabelecida com base no GPS Ideológico ${ }^{6}$, do qual selecionamos os 102 políticos mais voltados à esquerda, e os 102 mais à direita. Para cada usuário da amostra calculamos a proporção entre o número de políticos da lista da direita presentes nos followings do usuário pelo somatório dos políticos de esquerda e direita também presentes nos followings. Para ajustar o cálculo da medida de polarização política dos casos onde o usuário não segue políticos de direita nem esquerda, iniciamos a contagem com o valor 1 para cada lado. Assim, o valor 50\% indica usuários politicamente neutros, pois não seguem políticos ou seguem em igual proporção. Quanto maior (ou menor) o valor da métrica, mais orientado à direita (esquerda) é o usuário.

Foram também construídas redes representando cada movimento, onde cada nó representa um usuário, ligado a seus followings por arestas dirigidas. Para analisar cada rede, calculamos medidas globais, que mensuram a complexidade da rede (e.g. grau, caminho mais curto médio, diâmetro, coeficiente de clusterização). Posteriormente, foi feita a detecção de comunidades (i.e. subgrupos) dentro de cada rede, utilizando o software Gephi ${ }^{7}$. Para cada comunidade encontrada, foram calculadas as mesmas medidas já descritas, bem como a centralidade de proximidade (responsável por influenciar mais diretamente as redes) e de intermediação (responsável por espalhar as informações ao longo da rede) dos nós. Utilizamos os mesmos políticos para avaliar a polarização das comunidades resultantes.

\subsection{Aspectos Psicológicos Derivados de Características Linguísticas}

Trabalhos como [Demszky et al. 2019; De Choudhury et al. 2016] exploraram traços psicológicos relacionados com movimentos sociais ou posicionamentos políticos, derivados do uso diferenciado de categorias linguísticas específicas. Eles exploram a premissa de [Tausczik and Pennebaker 2010] de que as funções das palavras, representadas em categorias pelo LIWC, refletem estados psicológicos básicos, tais como estados emocionais e estilos de pensamento, etc. Investigamos cada movimento de acordo com os seguintes aspectos psicológicos, atrelados a sua relação com as categorias linguísticas:

- Coesão e união: investigamos se os movimentos têm traços de união/coesão distintos. Os experimentos de [Tausczik and Pennebaker 2010] indicam que a utilização de palavras da classe nós por indivíduos em um grupo denota aspectos de coesão, e que uma maior utilização da classe concordância pode sinalizar um maior consenso. Outros trabalhos associam estas classes à noção de pertencimento e envolvimento [Demszky et al. 2019; De Choudhury et al. 2016].

- Estados Afetivos: Pessoas tendem a expressar emoções negativas ou positivas em maiores proporções de acordo com a percepção do contexto no qual se inserem, utilizando assim mais palavras positivas/negativas [Harb et al. 2019; Tausczik and Pennebaker 2010]. Segundo [Demszky et al. 2019], a expressão de estados emocionais constitui um nível semântico relevante à polarização, e

\footnotetext{
${ }^{6}$ http://temas.folha.uol.com.br/gps-ideologico/

${ }^{7}$ https://gephi.org/
} 


\begin{tabular}{lllll} 
& \multicolumn{3}{c}{ Tabela I. } & Tópicos por Movimento \\
\hline Tópico & Tweets & Usuários & Densidade & Palavras - Cloroquiners \\
\hline 0 & 12855 & $\mathbf{5 3 5 2}$ & 2,40 & trabalhar, vamos, povo, quer, dinheiro, trabalho \\
1 & 11351 & 4009 & 2,83 & risco, país, quarentena, mundo, casa, fome \\
2 & $\mathbf{1 5 2 9 3}$ & 3984 & $\mathbf{3 , 8 3}$ & presidente, bolsonaro, brasil, tudo, pronunciamento, deus \\
\hline Tópico & Tweets & Usuários & Densidade & Palavras - Quarenteners \\
\hline 0 & 4634 & $\mathbf{2 1 5 9}$ & 2,14 & bolsonaro, mortes, governo, saúde, campanha, todos \\
1 & $\mathbf{5 1 0 5}$ & 1715 & $\mathbf{2 , 9 7}$ & genocida, presidente, vírus, urgente, carreata, povo \\
2 & 4799 & 2027 & 2,36 & brasil, parar, agora, vamos, bozo, contra \\
\hline Tópico & Tweets & Usuários & Densidade & Palavras - Neutros \\
\hline 0 & 8064 & 2804 & 2,80 & melhor, mãos, sempre, água, cuide, lave \\
1 & 10159 & 2944 & 3,45 & casa, ficar, pode, fica, saúde, ajudar \\
2 & 9851 & 3114 & 3,16 & isolamento, social, ainda, pessoas, vírus, corona \\
3 & 9755 & $\mathbf{5 1 0 5}$ & 1,91 & brasil, covid, bolsonaro, país, casos, mortes \\
4 & 12292 & 3655 & 3,36 & todos, vamos, vida, deus, passar, amor \\
5 & 12229 & 3148 & 3,88 & quarentena, aqui, fazer, tudo, agora, amigos \\
6 & $\mathbf{1 3 2 0 7}$ & 3159 & $\mathbf{4 , 1 8}$ & semana, quero, noite, coisas, sair, música \\
7 & 7930 & 4309 & $\mathbf{1 , 8 4}$ & hoje, live, jorge, galera, instagram, parabéns
\end{tabular}

pode auxiliar na deteç̧ão de níveis ideológicos. Selecionamos as categorias de emoções positivas e negativas do LIWC, assim como as subclasses negativas de raiva, ansiedade e tristeza.

- Sofisticação cognitiva: sofisticação cognitiva é um conceito relacionado com o quão sofisticado é o pensamento abstrato ou conceitual de alguém, sendo tipicamente associado com uma maior capacidade de discernimento entre conteúdo verdadeiro ou falso. Estudos preliminares afirmam que uma menor sofisticação cognitiva tem um impacto maior que a ideologia em termos de percepções equivocadas sobre a pandemia da COVID [Pennycook et al. 2020]. Assim como [Slatcher et al. 2007; De Choudhury et al. 2016], utilizamos de forma agregada as classes exclusividade, conjunções, preposiçôes e mecanismos cognitivos, que compõem os aspectos de cognição presentes em uma linguagem [Tausczik and Pennebaker 2010].

- Preocupações pessoais: para alinhar os tópicos encontrados com as preocupações pessoais dos indivíduos de cada movimento, utilizamos as subcategorias da classe Preocupações Pessoais do LIWC, i.e. trabalho, realizações, dinheiro, lazer, casa, religiosidade e morte.

Para realizar esta análise, utilizamos uma versão em português do $\mathrm{LIWC}^{8}$, contando para cada tweet as palavras de cada categoria considerada (0 se ausente, 1 se presente). O objetivo é analisar as implicações que os percentuais de utilização das classes selecionadas para o estudo trazem para a caracterização da identidade dos movimentos analisados nas diferentes dimensões. Para analisar a significância da diferença de percentuais de uso tanto entre movimentos, como par a par, foi executado o teste estatístico não paramétrico qui-quadrado com nível de significância de $95 \%$.

\section{ANÁLISES}

4.1 Q1: Há diferença nos assuntos comentados dentro de cada movimento?

Embora o assunto central dos 3 movimentos seja o isolamento social, observamos diferenças específicas dentro de cada grupo. A Tabela 4.1 mostra para cada grupo os tópicos encontrados, a quantidade de tweets e de usuários abordando cada tópico, junto com as 6 palavras mais representativas de acordo com o peso para associar ao tópico. A partir da observação das palavras de maior peso e inspeção manual dos tweets relacionados, pode-se conjecturar sobre os assuntos centrais de cada tópico.

Para o grupo dos Cloroquiners, os tópicos são centrados em economia e seus efeitos, ou apoio ao presidente. O Tópico 0 aborda a necessidade de volta ao trabalho, o Tópico 1 aborda e compara o isolamento social no país e no mundo, e o Tópico 2 aborda assuntos políticos de apoio ao presidente. Os Quarenteners manifestam a oposição à campanha governamental. O Tópico 0 critica a campanha do governo, o Tópico 1 exprime preocupação com ações do presidente e seus apoiadores e o Tópico 2 enfatiza o isolamento social para frear a proliferação do vírus. Nota-se que em ambos movimentos os tópicos de apoio/rejeição ao presidente e suas ações são aqueles que concentram a maior densidade de postagens por usuário, demonstrando existir membros politicamente engajados em cada grupo.

O grupo Neutro é o mais diversificado, centrado no vírus e no isolamento. O Tópico 0 trata da higienização das mãos. Tópicos 1 e 2 reforçam a importância do isolamento e os riscos em caso de

\footnotetext{
${ }^{8}$ http://www.nilc.icmc.usp.br/portlex/index.php/pt/projetos/liwc
} 


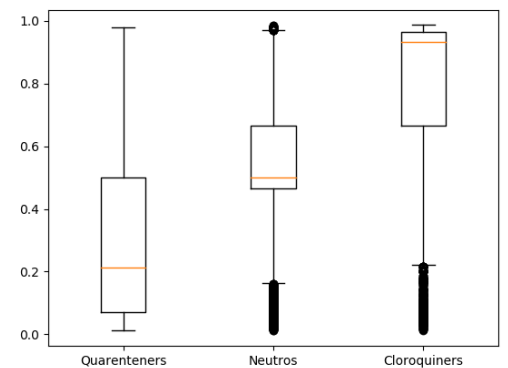

Fig. 1. Distribuição da orientação política nos movimentos

Tabela II. Propriedades dos Movimentos e de sua Comunidade Polarizada

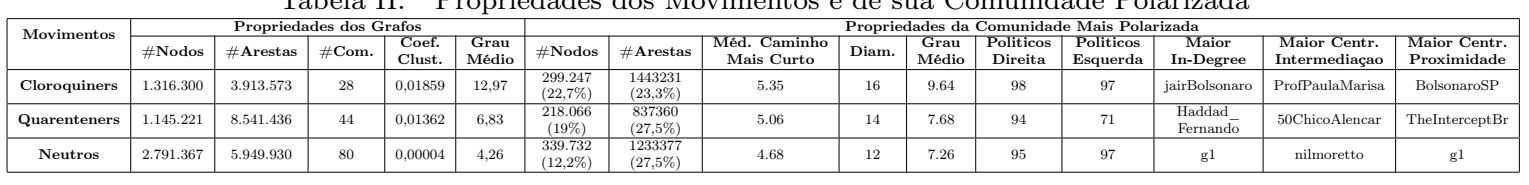

desrespeito. O Tópico 3 é relativo aos dados da pandemia, principalmente dentro do país, e às ações de combate do governo. O Tópico 4 concentra mensagens de esperança e positividade. Tópicos 5 e 6 abordam consequências do isolamento na rotina. O Tópico 7 trata de entretenimento virtual. Tópicos que abordam o isolamento $(1,5$ e 6$)$ são os que concentram maior número de postagens, enquanto que o entretenimento (6) tem os usuários mais engajados, confirmando a neutralidade deste grupo.

\subsection{Q2: Há diferença na polarização política dentre os movimentos?}

O boxplot da Figura 1 mostra a distribuição da métrica de polarização em cada movimento. Claramente o movimento dos Cloroquiners é altamente polarizado à direita. Com mediana de 92,3, este grupo concentra metade dos usuários da amostra acima deste percentual e apenas 25\% (Q1) tem índice menor que 66,29. Usuários com índice de polarização abaixo de 21,5 são considerados outliers.

Os Quarenteners têm caráter ideológico mais heterogêneo, com índices de polarização variando entre 0,6 e 97,3 (Min/Max). Dada a amplitude, não há outliers. Mesmo assim, 75\% dos usuários (Q3) possui orientação política mais à esquerda (49,9 ou menos), e 50\% dos usuários abaixo de 21 (mediana).

Finalmente, o movimento Neutro tem uma distribuição similar de usuários em cada um dos lados, com mediana de 50\%. Pelo menos 25\% (Q1) dos usuários têm índice de polarização igual ou inferior a 46,25, e 75\% dos usuários (Q3) têm valor menor ou igual a 66,3. Os valores min/max são respectivamente 16,4 e 96,5, sendo valores inferiores/superiores a estes considerados outliers.

A Tabela II mostra os resultados da análise das comunidades detectadas em cada movimento, pela análise dos grafos dos followings. Nota-se pelos coeficientes de clusterização que a probabilidade dos nodos formarem comunidades nos grafos dos Cloroquiners e os Quarenteners é maior comparados à rede dos neutros. Comparados a dos Quarenteners, a rede dos Cloroquiners tem menos comunidades, é mais fechada e densamente conectada. Examinando as comunidades de cada movimento, buscou-se aquelas com o maior número de políticos dentre os considerados. Notou-se que nos três movimentos existe sempre uma comunidade que concentra estes políticos em igual proporção direita/esquerda, e que as demais tipicamente não envolvem estes políticos.

As propriedades das três comunidades mais polarizadas de cada movimento também estão destacadas na Tabela II. A comunidade polarizada dos Cloroquiners (22,7\% destes usuários) é também a maior dentre todas as comunidade polarizadas, com os usuários mais conectados, e maior alcance. No caso dos Quarenteners, encontramos duas comunidades polarizadas. A mais polarizada (19\% destes usuários) envolve quantidades significativas de políticos de esquerda/direita, enquanto que uma segunda $(12,4 \%)$ envolve apenas políticos de esquerda (28), denotando que os usuários politizados deste movimento se engajam de formas diferentes. Comparativamente, a proximidade média dos usuários da comunidade mais polarizada dos Quarenteners e dos Cloroquiners é parecida, mas o alcance da rede é menor se comparados os diâmetros. Para os Neutros, a comunidade polarizada representa 12,2\% deste 
KDMiLe - Symposium on Knowledge Discovery, Mining and Learning - Applications Track

\begin{tabular}{|c|c|c|c|c|c|c|c|c|c|}
\hline Dimensão & Categoria & Neutros & Cloroq. & Quarent. & Dimensão & Categoria & Neutros & Cloroq. & Quarent. \\
\hline Senso de & nós & 0.06 & 0.08 & 0.04 & Emoções & raiva & 0.16 & 0.20 & 0.23 \\
\hline grupo & concordância & 0.06 & 0.09 & 0.06 & & tristeza & 0.21 & 0.20 & 0.20 \\
\hline Preocupações & trabalho & 0.34 & 0.41 & 0.29 & & ansiedade & 0.10 & 0.12 & 0.12 \\
\hline \multirow[t]{6}{*}{ Pessoais } & dinheiro & 0.25 & 0.27 & 0.23 & & em. negativa & 0.39 & 0.41 & 0.44 \\
\hline & lazer & 0.25 & 0.13 & 0.12 & & em. positiva & 0.57 & 0.51 & 0.41 \\
\hline & casa & 0.19 & 0.06 & 0.06 & Complexidade & exclusividade & 0.50 & 0.50 & 0.50 \\
\hline & saúde & 0.18 & 0.14 & 0.13 & Cognitiva & conjunção & 0.62 & 0.54 & 0.55 \\
\hline & morte & 0.05 & 0.07 & 0.08 & & preposição & 0.80 & 0.65 & 0.67 \\
\hline & & & & & & mec. cognitivos & 0.92 & 0.86 & 0.86 \\
\hline
\end{tabular}

grupo, com usuários mais próximos entre si e menor alcance da informação. Nota-se que os nodos com maior número de conexões nos Quarenteners/Cloroquiners correspondem aos adversários políticos nas eleições, enquanto que nos Neutros, a um jornal. Os maiores responsáveis por propagar notícias (centralidade de informaçao) têm viés político: uma youtuber seguidora de Olavo de Carvalho e um político de esquerda. Enquanto os Cloroquiners têm como maior fonte de informação (centralidade de proximidade) um político, nos demais grupos este papel é atribuído a mídias formais.

Pode-se concluir que os movimentos dos Cloroquiners e Quarenteners são efetivamente polarizados, sendo a tendência ideológica do primeiro orientada à direita, e do segundo orientada à esquerda, mas de forma menos acentuada e mais diversa. Os padrões de engajamento no Twitter mostram os Cloroquiners como uma comunidade mais fechada e próxima, centrada no repasse de informações via redes sociais, enquanto que os Quarenteners apresentam comportamentos de oposição mais diversos.

\subsection{Q3: Os movimentos apresentam aspectos psicológicos distintos?}

Para verificar as semelhanças e diferenças para o uso das palavras em termos de aspectos psicológicos, começamos aplicando o teste qui-quadrado nas 64 classes linguísticas do LIWC para comparar as diferenças nos 3 grupos. Todas as classes são significativamente distintas exceto uma. O teste para o par Cloroquiners/Quarenteners não resulta em diferenças em 19 classes; 3 classes no par Quarenteners/Neutros e 2 no par Cloroquiners/Neutros. A semelhança no uso de cerca de $30 \%$ das classes do LIWC entre Cloroquiners e Quarenteners mostra uma considerável tendência a construções linguísticas parecidas nos movimentos polarizados. A Tabela III apresenta os percentuais de uso das categoria utilizadas para investigar 4 aspectos psicológicos, discutidos a seguir:

- Coesão: A classe nós é significativamente mais presente nos tweets dos Cloroquiners, um indício de que este movimento possui maior coesão. O maior uso percentual da classe concordância nos Cloroquiners é significativo, e reitera a ideia de que seus indivíduos possuem maior senso de grupo. Não há diferenças significativas no percentual de uso de concordância entre Quarenteners e Neutros. Assim, temos evidências de que os Cloroquiners são um movimento marcado pela coesão e união.

- Emoções: Enquanto os Quarenteners têm o maior percentual de uso de palavras na categoria Emoções Negativas e menor em Emoções Positivas, os Neutros têm comportamento oposto (diferenças de 5 e 15,21 pontos percentuais, respectivamente). Cloroquiners seguem a mesma tendência dos Quarenteners, mas em percentual levemente menor. Quando examinadas as subcategorias negativas, Quarenteners expressam significativamente mais raiva que Cloroquiners, e percentuais estatisticamente similares de ansiedade e tristeza. Estes são indícios de que o grupo Neutro é o menos alheio a relatos traumáticos, e que as emoções positivas são confirmadas pelos diferentes tópicos discutidos pelo grupo na Seção 4.1. Nos movimentos polarizados, os maiores percentuais de emoções negativas contribuem à ideia da descrição de eventos traumáticos como forma de persuasão à ideia de seu grupo, ou simplesmente estar ligado ao pessimismo das ideias defendidas (condução das ações de controle da pandemia nos Quarenteners, e economia do país nos Cloroquiners).

- Sofisticação Cognitiva: considerando as quatro categorias utilizadas para descrever este aspecto, nota-se não haver diferença significativa no uso da categoria exclusividade entre os movimentos. O movimento Neutro apresenta os maiores percentuais de uso das outras três classes (mecanismos cognitivos, conjunções e preposições). Entre Cloroquiners e Quarenteners, apenas a diferença no uso de preposições é estatisticamente significativa. Pode-se concluir que o grupo de Neutros consegue expôr suas ideias em tweets com narrativas mais coerentes, complexas e concretas comparadas aos 2 movimentos politicamente polarizados, os quais possuem índices semelhantes para Sofisticação Cognitiva. Estes achados estão consistentes com [Pennycook et al. 2020], que relata que a ideologia 
não está relacionada com as crenças sobre a COVID, e sim à sofisticação cognitiva.

- Preocupações Pessoais: a utilização das classes desta dimensão do LIWC confirmaram os tópicos vistos na Seção 4.1: trabalho e dinheiro têm maiores percentuais entre Cloroquiners; lazer, saúde e casa em Neutros; e Morte mais ligado aos Quarenteners, ainda que em percentuais próximos.

Conclui-se que os movimentos polarizados diferem em termos de Preocupações Pessoais e Coesão de grupo, mas mostram-se mais próximos quando comparados com os Neutros nos aspectos envolvendo Emoções e Sofisticação Cognitiva. A negatividade fornece evidências que o posicionamento é marcado pelo descontentamento, e que a baixa sofisticação cognitiva influencia mais a percepção sobre a pandemia que a orientação política [Pennycook et al. 2020].

\section{CONCLUSÃO E TRABALHOS FUTUROS}

Caracterizamos neste artigo três movimentos distintos no Twitter relacionados à COVID. Para esta análise, propusemos um framework que unifica distintas dimensões desta caracterização. Além da modelagem de tópicos, propõe uma métrica para mensurar a polarização política, a análise da estrutura social usando métricas topológicas de redes, bem como a caracterização de aspectos psicológicos baseada no uso da linguagem. Este mesmo framework de análise pode ser usado para investigar as implicações do posicionamento político em diferentes temas, tais como educação ou meio ambiente.

Comprovamos que os pontos de vista são específicos à polarização política. O grupo mais polarizado e coeso é dos Cloroquiners, fortemente de direita. Quarenteners são polarizados à esquerda. Os temas que os diferem refletem a polarização no apoio/rejeição ao presidente ao estabelecer o dilema entre vidas e economia. Ambos são semelhantes em termos de sofisticação cognitiva e emoções negativas, apresentando evidências de que seu ponto de vista origina-se no descontentamento, e que a baixa sofisticação cognitiva é mais influente na percepção sobre a pandemia do que a orientação política.

Pretendemos evoluir o framework de análise e sua utilização em outros cenários. Os tópicos incluem o detalhamento do comportamento das comunidades mais polarizadas e sua diferença em relação aos usuários do mesmo grupo; expansão dos aspectos psicológicos estudados e sua relação com as categoria linguísticas; investigação dos aspectos psicológicos preditivos da polarização, entre outros.

\section{REFERÊNCIAS}

Blei, D. M., NG, A. Y., And Jordan, M. I. Latent dirichlet allocation. vol. 3, pp. 993-1022, Mar., 2003.

De Choudhury, M., Jhaver, S., Sugar, B., and Weber, I. Social media participation in an activist movement for racial equality. In Proc. of the 10th Intl. Conf. on Web and Social Media (ICWSM). pp. 92-101, 2016.

Demszky, D., Garg, N., Voigt, R., Zou, J., Shapiro, J., Gentzkow, M., And Jurafsky, D. Analyzing polarization in social media: Method and application to tweets on 21 mass shootings. In Proc. of the 2019 Conf. of the North American Chapter of the Association for Computational Ling.: Human Language Technologies. pp. 2970-3005, 2019.

Garimella, V. And Weber, I. A long-term analysis of polarization on twitter. In Proc. of the 11th Intl. Conf. on Web and Social Media (ICWSM). pp. 528-531, 2017.

Harb, J., Ebeling, R., And Becker, K. Exploring deep learning for the analysis of emotional reactions to terrorist events on twitter. Journal of Information and Data Management 10 (2): 97-115, 2019.

Ordun, C., Purushotham, S., and Raff, E. Exploratory analysis of covid-19 tweets using topic modeling, umap, and digraphs. arxiv:2005.03082, 2020.

Pennycook, G., McPhetres, J., Bago, B., And Rand, D. Predictors of attitudes and misperceptions about covid-19 in canada, the UK, and the USA. doi:10.31234/osf.io/zhjkp, 2020.

Röder, M., Both, A., And Hinneburg, A. Exploring the space of topic coherence measures. In Proceedings of the eighth ACM international conference on Web search and data mining. ACM, pp. 399-408, 2015.

Sha, H., Hasan, M. A., Mohler, G., and Brantingham, P. J. Dynamic topic modeling of the covid-19 twitter narrative among u.s. governors and cabinet executives. arxiv:2004.11692, 2020.

Slatcher, R., Chung, C., Pennebaker, J., and Stone, L. Winning words: Individual differences in linguistic style among u.s. presidential and vice presidential candidates. Journal of Research in Personality 41 (1): 63 - 75, 2007.

TAusczik, Y. R. And Pennebaker, J. W. The psychological meaning of words: Liwc and computerized text analysis methods. Journal of Language and Social Psychology 29 (1): 24-54, 2010. 